\title{
XI. A sensitive-strip spectropolariscope
}

\section{Professor D.B. Brace}

To cite this article: Professor D.B. Brace (1903) XI. A sensitive-strip spectropolariscope, Philosophical Magazine Series 6, 5:25, 161-170, DOI: 10.1080/14786440309462907

To link to this article: http://dx.doi.org/10.1080/14786440309462907

$$
\text { 册 Published online: } 15 \text { Apr } 2009 .
$$

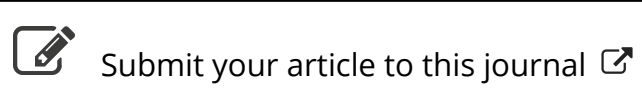

\footnotetext{
Џll Article views: 2
}

Q View related articles ¿

Citing articles: 2 View citing articles $\square$ 
considering, which is rapidly damped, the time for a halfoscillation would increase as the discharge died away, a result experimentally obtained and published in a letter written to 'Nature' by the author in August 1900.

An interesting application of the method is the determination of the deflexion that would be attained by a ballistic galvanometer when a discharge passes through it which cannot be assumed to have ceased flowing before the needle has moved. In this case the value of $\frac{\mathrm{E}_{0}}{\mathrm{R}}$ would be variable, and the subtraction would have to be repeatedly performed from a variable point.

In conclusion I wish to express my indebtedness to Mr. G. W. Worrall, B.Sc., for working out some of the values of the self-induction under varying currents which have been used in plotting the curves given above.

University College, Liverpool.

\section{A Sensitive-strip Spectropolariscope.! By Professor D. B. Brace *.}

TTHE conditions for maximum sensibility of the eye in. making comparisons with the polariscope are the same as those of a photometer. The entire displacement, except in technical work, of photometric comparisons with ordinary light by spectral photometric observations, which the growing demands in photometry for exact data on specific colours have produced, illustrates the corresponding condition in polariscopic work. The importance, both theoretical and practical, of determining, on the one hand, the relative distribution of intensities subjectively and objectively in any radiant and, on the other, tho relative transmission of any absorbent for different periodicities, bas necessitated the highest instrumental refinements.

It is now possible to obtain settings for the mean spectral colours with a probable error of less than one-fifth per cent. $\dagger$ and on some occasions with a carefully trained eye as low as one-tenth per cent. This has been brought about by inproving the viewing screen so that the bounding lines between the comparison fields should be perfectly sharp and vanish with equal illumination of the fields, the colour of course becoming the same over the entire field of view. In

* Communicated by the Author. Read before the American Physical Society at its Pittsburg meeting, July 1902.

$\dagger$ Phil. Mag. [5] xlviii. p. 420 (1899).

Phil. Mag. 8. 6. Vol. 5. No. 25. Jan. 1903. 
the spectrophotometer the best results have been obtained by means of a silver strip. The photometer is subject to the further restriction in its use of measuring the quantities of light. This element, as a difficulty, is really negligible in the polariscope, but the former difficulty does not seem yet to have been overcome.

In a polariscope it is necessary to vary the so-called sensibility as the amount of light varies. This condition should obtain too for all colours. For the greatest efficiency, the polarizing and analysing elements should not displace the ray when placed in its path, as this would generally affect the position of the image for any one wave-length. The bounding lines between the fields should also vanish for any colour and sensibility and that too whether a broad or a narrow radiant is the source of light. Some of these conditions have been realized in the types already devised, but no one embodies them all.

In the biquartz of Soleil a vanishing line is partially realizable, but as the tint of passage is used white light is required; and further the sensibility cannot be varied. In a double rotary element, such as that of Poynting rotating. differentially in the same direction, a vanishing line is also partially realizable; but its use over a finite portion of the spectrum of sufficient breadth to give a proper intensity will make initial settings of different tints in the different parts of the field, owing to the differential rotation. The neutral position of the analyser will also vary in different parts of the spectrum for similar reasons. This form and the modified form of the biquartz with a small angle of rotation which is used for monochromatic light do not admit of a practical variation in the sensibility. Furthermore, the added rotation of any medium under examination increases the differential rotation of one portion of the field over the other, thus intensifying the difference of tint in the two fields, which diminishes the sensibility in setting. In the polariscopes of Savart, Babinet, Nodot and others a vanishing and a displacement of interference-bands take place. The settings which can be made with these forms are far less accurate than those already mentioned and in some of them very fatiguing to the eye. In the system of Laurent, with a halfwave plate, much used in saccharimetry at the present time, a vanishing line is attainable and the sensibility can be varied, but light of only one colour can be used. In the half-shade polarizer of Jellet any colour may be used, but it is impossible to vary the sensibility and also to eliminate the dark line between the two halves of the field. In the half-shade 
polariscope of Lippich, in which the field is either bisected or trisected, we have the most adaptable and sensitive instrument which has been devised. This form embodies the very essential feature of a variable sensibility, and, with a sufficiently broad source, a nearly vanishing line is possible. However, in most forms of nicols, since it is the extraordinary ray which is used, there is a displacement of its path. 'This of course can be avoided if the ray enters normally a nicol with the optic axis in the intersection of its face with its diagonal plane. Most nicols are also found to be slightly prismatic. The ray will usually receive a further displacement on its. passage through the second nicol which covers a part of the field of the first one. The two ray systems thus give separate images, which, in spectral work, is a serious difficulty in obtaining a perfect match over the field. In setting for any one colour a rotation of either polarizer or analyser should not displace the image. To avoid this, care must be exercised to eliminate the errors due to displacement mentioned above. It does not seem possible to produce a vanishing line between the different parts of the field except with a source of sufficient breadth. With such a radiant at a focus conjugate to the image at the analyser, when the lens is just before the polarizing nicol, there is a great number of bundles of rays which thus produce uniform illumination over the entire field. When, however, the source is very narrow and we have approximately a single bundle of rays, the case is different and we generally observe a decided dark or bright line between the fields, which greatly reduces the delicacy of perception of the eye. This is evidently an irremedial effect since the polished side of the second nicol, usually several centimetres long, will reflect internally or externally any rays striking it, and it is thus impossible to obtain a continuous system of rays across this bounding face, particularly if the rays within it are displaced as described above. In several of the most sensitive half-shade combinations obtainable this defect existed to a greater or less extent. In spectral work, except with bright-line radiants or with absorption cells which never give sufficient homogeneity, a narrow source or slit is essential. Hence the Lippich form of instrument does not seem to be available for general colour observations over successive portions of the spectrum. The amount of light obtainable from a bright-line radiant, e.g. an Aron's lamp, was found to be far below that obtained from the direct rays of the same colour from the sun after passing through a spectral system as is usually desirable, e.g. absorbing media which have anomalous M 2 
rotary effects. Other defects usually found are imperfect polishing and figuring of the nicols themselves. These are usually only observed in work with spectral colours from a narrow source giving approximately a single bundle of rays. The scratches and ridges here become at once evident and any variation in the curvature of the face manifests itself in a variation of intensity of the field, producing a shaded or mottled appearance. "This is a well-known appearance * in spectrophotometry when the ray system is not coaxial with the lenses or when their curvature is not uniform.

In the nicol prism a total reflexion of the ordinary ray takes place at the internal diagonal faces which are cemented with balsam or with evaporated turpentine-oil or linseed-oil, whose indices are less than that for the ordinary ray. This allows a part when balsam, or practically all when these oils are used, of the extraordinary ray to pass through. The reflected ray is absorbed by the sides of the nicol but not completely. When an intense source like the sun is used the diffused light is so large as to produce a decided effect on the bue when colours of very low intensity reach the eye. This of course is a difficulty which the optician can eliminate by proper casing and diaphragming.

It is not a difficult matter mechanically and optically to reverse the conditions of reflexion with the optical media now available. For example, we have as the index of the ordinary ray of spar for the D-line 1.6584 , while for the same line the value for $\alpha$-monobromonaphthaline is 1.6582 . Schott and Co., of Jena, also cast a glass the value of which is $n_{p}=1 \cdot 6527$. The dispersion of each of these is of course different, but for the mean colours of the spectrum the indices are almost the same. It has seemed heretofore impossible to obtain glass so free from strain as to produce no observable depolarization. The use of liquids, however, has been found perfectly practicable and efficient. Solutions of carbon disulphide may be used giving almost any convenient index from a low value with balsam to an index greater than $1 \cdot 7_{\mathbf{D}}$ with phosphorus.

The well-known optical fluid $\alpha$-monobromonaphthaline is, however, much superior, as it remains nearly colourless if sealed up and not subjected to actinic action by continuous exposure to light. Its index is the same as that of spar for the mean portions of the spectrum. If now the cementing film and the prisms of spar are interchanged, we should have an interchange of the extraordinary and ordinary rays. Thus a plate of spar placed diagonally at the proper angle within a

* Tuckerman, Astrophys. Journ. xvi. p. 145. 
cylinder of glass or of this fluid would totally reflect the extraordinary ray and transmit almost completely, and without any displacement as is usually the case in nicols, the ordinary ray. A second cylinder containing a narrow thin strip of the spar would give us a bisected or trisected field with a vanishing line.

In cutting* the spar for both the first polarizer and for the sensitive strip, two directions in the crystal have been tried. In the first system the longest axis of the plate lies in a principal plane through the optic axis, making an angle of $70^{\circ}$ with it. The first plate was cut about $2 \mathrm{~mm}$. thick and in the form of an ellipse with its edges ground away so as to fit within the cylindrical cell containing the liquid. The dimensions of this ellipse were $44 \mathrm{~mm}$. by $15 \mathrm{~mm}$. over one face, thus giving a clear circular aperture of $15 \mathrm{~mm}$. with an angle of incidence of $70^{\circ}$. The sensitive strip was cut in the same plane but only $5 \mathrm{~mm}$. by $44 \mathrm{~mm}$., and rectangular in shape. The thickness was $0.15 \mathrm{~mm}$. and its lateral edges perfectly sharp and square with its faces. Experience with plates of these dimensions and thicknesses show that they may be safely reduced to $0.1 \mathrm{~mm}$. or less in thickness. In the second system the plates were all cut rectangular and to the same dimensions as those of the first. Their direction. within the crystal, however, is different, their faces being principal planes through the optic axis which is perpendicular to the longest edge or length of the plate. More difficulty was experienced in grinding these as they fractured more readily and a thickness of $0.5 \mathrm{~mm}$. was found practicable. More material was also required for this system.

In order to obtain total reflexion of the extreme red rays, an angle of incidence of $65^{\circ}$ is necessary. To provide for total reflexion of a cone of rays, $68^{\circ}$ to $71^{\circ}$ is required. The angle of incidence adopted is $70^{\circ}$. This gives a length of $40 \mathrm{~mm}$. for a cell of $15 \mathrm{~mm}$. clear aperture. The cost of a plate itself is about one-third that of a nicol of the same aperture. The ellipse or first plate is mounted within a cell of about twice the diameter of the aperture in order to provide for sufficient fluid and effective diaphragming. There are five of these diaphragms fixed to a concentric tube and they are slightly slotted along a diagonal so as to admit and retain the polarizing plate. This latter is cemented slightly at its ends to the blackened tube with fish-glue (Le Page's), mixed in four parts with two of glycerine and five of water. Care is exercised in avoiding any strain as depolarization will

* The plates were cut and polished by Bernhard Halle, Steglitz-Berlin. 
be present under the slightest effect induced by the cement or otherwise. Thin cover-glasses are carefully selected and cemented on the brass caps or end-pieces which screw into the tube. No moisture or acid used on the metal must be allowed to reach the plate. The liquid itself shows no action on spar. As it is quite volatile the cell is closed tight, with a bubble of air remaining in to compensate for expansion. The sensitive strip may be mounted in the same cell, but in order to vary the sensibility one of the plates must be capable of a slight displacement. The mechanical difficulties are greater in such a system ; but higher efficiency is possible. On the other hand, greater simplicity is obtained by mounting the strip in a separate cell and then placing the two cells in a common tube and rotating the first, as is usually done in the mounting of the Lippich system. With such thin strips of spar, of course care must be exercised in mounting them so that they vill not fracture or suffer strain. This was done by cutting away a slight portion in the inner circumference of the caps of the tube and cementing the ends of the strip to these with the cement mentioned. Thin cover-glasses were then cemented over the ends of the blackened brass cell and the liquid inserted through a small opening which was later sealed up. However, such a strip might at the outset show no trace of depolarization, and later not give perfect blackness owing, manifestly, to the setting of the cement and the induced strain in the strip. This was obviated by cementing one end only and leaving the other slightly free within retaining contacts. When these precautions are taken and the system examined in the ordinary way with analyser and telescope, the surprising sharpness between the fields is at once observed and, with a neutral setting, as perfect a vanishing line is obtained as with the most delicately adjusted strip in the best spectrophotometer. This system, properly mounted with parallel end-plates, when placed in any ray system will give a single image and cause no deviation of the axis of the ray system. The sensibility of the system can be varied easily and a vanishing line obtained. It may also be used for any colour and the neutral setting will not vary for different parts of the spectrum. When the liquid is freed from suspended particles by filtration no diffused light emanates from the polarizer and a perfectly black field is obtainable even with direct sunlight. These conditions are not so quickly obtained as with the system of Lippich, but when they are, a greater sensibility is possible. For a single bundle of rays or such as would emanate from a narrow source or slit, it is evident that, on account of the narrow edge of the 
strip as compared with the long polisbed side of the second nicol in the lippich form, a much more continuous system of rays is possible and hence a far sharper bounding or a vanishing line between the fields.

Various systems have been tried for obtaining different monochromatic light of sufficient intensity. As mentioned above, the intense system of Aron gives only definite wavelengtbs and hence cannot be used for all purposes. Any one of the mercury lines was also found to be much less intense than a corresponding portion of the spectrum sufficiently narrow to give, within the limits of observation, the same rotation of all its components in the experiments tried. Absorption cells are not sufficiently monochromatic for many observations and are of course restricted in the colours available. Except in the sensitive-strip system here described, a broad diffusing source is necessary. Here too sunlight gives the best results; but in order to use it as such, a diffusing plate, e.g. a groundglass or milk-glass plate, must be inserted. This is found to reduce the available light by 95 per cent. to 98 per cent. Owing to the sensibility of the system any arbitrary spectral arrangement will not suffice. No light should be allowed to enter the polarizing system which is not used in the immediate observations. The spectral system adopted must fulfil these conditions. The most obvious arrangement and one used largely is to place a slit at a focus conjugate to the analyser and with the lens immediately at the polarizer, a dispersing prism being placed immediately before or after the lens. A spectrum is thus formed in the plane of the analyser and by a proper diaphragm or slit in this plane, the eye, aided by a telescope or not, will see the field illuminated with a uniform tint. This is owing to the fact that the retina is a conjugate focus to the field aperture, which is a colour radiant, and its lens converges all rays of different colours radiating from one point of the field (dispersing prism) to a common focus on the retina. If, however, a cell of absorbing substance, say for the green, is placed in the path of this beam, the very small amount of green which is transmitted may be largely neutralized by extraneous light or by the smallest fraction of red and blue which is diffused from the faces of the prisms or from internal reflexions of the system, which thus becomes a radiant. This has been found to give apparent rotations following some regular law predicted on entirely different grounds. To avoid these spurious results, the entire dispersion and separation of the colour should take place outside and beyond the polarizer. This of course may be attained in many ways, several of which have been tried, 
e.g. the dispersion may be obtained by a single optical system and the resulting spectrum formed in the plane of the slit abcve referred to as conjugate to the analyser. A suitable mirror before the prismatic system may be made to rotate any colour into this slit and maintain the system of rays used homocentric to any axis in the system. It is not easy, however, with such a system to obtain a uniform intensity over the entire field of view. The difficulty from diffusion referred to, however, is eliminated.

The system which has given thus far the best results is a modification of one devised by the writer for colour mixtures and used by Doubt* in his determination of the colour equations for different radiants. This is a reversing coloursystem consisting in reality of a double dispersing system. $A$ concave mirror whose radius of curvature is the focal distance of the cone of rays emerging from the prism and lens is placed in the focal plane. The rays of different wave-lengths strike it at the same anglo and hence are reflected back and recombined in the prismatic system and brought to a focus at the slit source. Evidently any portion of the spectrum would be recombined here. All screens with such a narrow slit were found to diffuse more or less the remaining colours of the spectrum, so that a single narrow mirror was used to reflect a corresponding portion of the spectrum, the remaining portion being allowed to pass on to the darkened walls of the room. After returning through the prismatic system where all rays were recombined, a mirror reflected the cone of rays through the polarizing system which come to a focus at the analyser. In this way fields of perfectly uniform tint with vanishing lines for a neutral setting were obtained, and, using the sun's rays, a sensibility for the mean colours greater than that which had previously been possible with the Lippich form using white light. The accompanying table (p. 169) gives a series of successive settings taken at random by Mr. Bates, Fellow in Physics, to whom is due much credit for the perfection of this arrangement and the elimination of the spurious effects in anomalous dispersive substances observed by the other experimenters.

These give the deviation of a single setting from the mean of from one two-hundred-and-fiftieth to approximately one twohundreth of a degree for these three wave-lengths.

In natural rotative substances this system needs no special mounting, but in the study of magnetic rotation it must be free from the action of the field. 'This has been accomplished,

* Phil. Mag. [5] xlvi. p. 216 (1898). 


$\begin{array}{lll}\text { C-line. } & \text { D-line. } & \text { E-line. } \\ .075^{\circ} & \cdot 095^{\circ} & \cdot 1575 \\ .075 & \cdot 095 & \cdot 1575 \\ .0775 & \cdot 100 & \cdot 1600 \\ .075 & \cdot 1025 & \cdot 1550 \\ .075 & \cdot 095 & \cdot 1550 \\ .075 & \cdot 0975 & \cdot 1550 \\ .070 & \cdot 0975 & \cdot 1575 \\ .075 & \cdot 0975 & \cdot 1575 \\ .075 & \cdot 0975 & \cdot 1525 \\ .075 & \cdot 1000 & -1550 \\ .075 & \cdot 095 & \cdot 1525 \\ & \cdot 095 & \\ & \cdot 095 & \\ & \cdot 095 & \end{array}$

when it is near the system, by housing it in a thick iron cylinder of suitable length which becomes at once the containing tube for the cells. Attention should also be called to the further fact that when the rotation is measured immediately by the analyser the different elements of the field sometimes show a slight difference in tint if the rotation is considerable. This is due to the fact that the light is not strictly homogeneous, and the different components are rotated by different amounts. This could be wholly or partially avoided by using a rotary compensator, e.g. quartz wedges.

For perfect adaptability to any wave-length there is no doubt that systems giving channeled spectra are preferable, but on this account only, as their sensibility is far less than the half-shade or sensitive-strip instruments. A rotation equal to the amount observable in the system described and referred to in the table above means a displacement of only one forty-thousandth of the distance between two black bands in a channeled spectrum. It is very doubtful indeed whether a setting in channeled spectra closer than one degree can be made with the same certainty as one two-hundredth of a degree with this system. This means one two-hundredth of a band, which ordinarily is very high indeed. However, some observers have claimed settings as accurate as from ten to three ove-hundredths of a degree or one six-thousandth of a band. Experienced observers in repeating such observations with similar apparatus have never been able to set, with certainty, closer than one or two degrees. Such a realizable sensibility would truly be an immense step in advance, as a 
channeled spectrum is really the only true representation of dispersive gradation. Until this can be unquestionably demonstrated, the use of the sun with the sensitive-strip system must be relied upon on account of its greater sensibility and adaptability for any spectral study.

Physical Laboratory,

University of Nebraska, Lincoln.

\section{Notices respecting New Books.}

Naturlehre. Von Dr. Alors LanNen. Mit 377 Figuren, einer Spectraltafel und 4 meteorologisehen Karten in Farbendruck. Wien: Verlag der Jos. Roth'schen Verlagsbuchhandlung. 1902. Pp. 377.

THE task of writing an elementary text-book of reasonable size which should contain a brief account of all the branches of physics (including the elements of astronomy) and chemistry is a someirbat formidable one, and we must congratulate the author on its successful accomplishment. Wisely abstaining from trying to confront his reader with an unmanageable mass of facts, the author makes a careful selection of such phenomena as serve to illustrate and bring home to the mind of the student the leading principles of modern science.

In spite of its elementary nature, the book is thoroughly up to date, and the interest of the reader is maintained by frequent references to the numerous practical applications of the principles dealt with in the book.

There is a peculiar interest attaching to this work. It has been written along the lines laid down by the Austrian Ministry of Education for the guidance of teachers in the State secondary schools or "gymnasia." A boy who has mastered the contents of this book has certainly obtained a good insight into physical science, and is splendidly equipped for the subsequent training in a technical college or university. One cannot help thinking how much easier the work of higher technical education would become in England, if only every secondary school were to put its pupils through some such course as that contained in the book under review.

\section{Proceedings of Learned Societies.} GEOLOGICAL SOCIETY.

[Continued from vol. iv. p. 520.]

May 28th, 1902.-Prof. Charles Lapworth, LL.D., F.R.S., President, in the Chair.

THE following communications were read:-

1. 'The Red Sandstone-Rocks of Peel (Isle of Man).' By William Boyd Dawkins, M.A., D.Sc., F.R.S., F.G.S., Professor of Geology in Owens College (Victoria University), Manchester.

The Red Sandstone Series, ranging along the coast from Peel to Will's Strand, is faulted into the Ordovician massif of the Isle of 